\title{
Desafiando la realidad laboral: \\ Programa de capacitación laboral para personas con discapacidad intelectual
}

\begin{abstract}
Glukman R, Yael.(1); Zamorano U,Kenia (2);Núñez C,Paula(3);Valderrama
G.,Marisol (4)

(1)Terapeuta Ocupacional. Licenciada en Ciencias de la Ocupación

Humana.(2)Educadora Diferencial con Mención en Discapacidad intelectual.

Licenciada en Educación. (3)Asistente Social. Licenciada en Trabajo Social con

Mención en Desarrollo Local.(4)Profesora en Educación Diferencial. Licenciada en

Educación. Escuela Especial Gabriela Mistral № 192

Contacto > > yglukman@hotmail.com,kensico@cvmail.cl,paanuca@hotmail.com, Dirección Postal de la Escuela Especial Gabriela Mistral № 192: Arturo Prat № 139

La Cisterna. Fono: 55832 66/527 6164 gmistral192@yahoo.com

Referencia > > Glukman R, Yael;Zamorano U,Kenia; Núñez,Paula, et al. Revista Chilena de Terapia Ocupacional.№5, Noviembre 2005.
\end{abstract}

\section{- Abstract}

This paper presents a Labor Program for the Intelectual Disability that is taking place at the Escuela Gabriela Mistral. Its goal is to favor the sociolaboral integration of its students inside the community. It tries to demonstrate that, even with low resources, an interdisciplinary working group may establish nets that will accept this people, thus allowing them to join the labor world in good conditions.

\section{Resumen}

Este artículo se propone presentar el Programa de Capacitación Laboral para Personas con Discapacidad Intelectual que se lleva a efecto en la Escuela Gabriela Mistral. Su objetivo es favorecer la integración sociolaboral de los alumnos en la comunidad. Se intenta demostrar que, aunque los recursos sean escasos, un equipo de trabajo interdisciplinario logra establecer redes que acojan a este sector de la sociedad y le permitan acceder al mundo laboral en buenas condiciones.

\section{- Introducción "}

La Escuela Especial No 192, Gabriela Mistral, ubicada en la comuna de La Cisterna de Santiago de Chile atiende, hace veintiséis años, a 250 niños y jóvenes con necesidades educativas especiales. La mayoría del alumnado proviene de comunas del sector sur de Santiago: La Cisterna, El Bosque, La Pintana y San Bernardo.

La escuela está organizada en torno a dos ejes educativos:

1. Programa de Educación Especial, con dos sub ejes:

a) Cursos básicos especiales para alumnos de 6 a 17 años. 
b) Programa de capacitación laboral, con dos Pretallares y tres Talleres Laborales, para alumnos de 18 a 26 años.

2. Programa de Cursos para integración, con tres sub ejes:

a) Cursos para integración de $1^{\circ}$ a $7^{\circ}$ básico.

b) Programa de preparación para exámenes libres para alumnos de 18 años en adelante a través del Departamento Provincial de Educación (Deprov) Sur.

c) Programa de escuela de recursos como apoyo a la escuela básica regular.

En el año 2004 se realizó en la Escuela un estudio de la realidad socioeconómica del estudiantado basado en datos de anamnesis, matrícula y entrevista con apoderados. Los resultados indicaron que la población escolar se estructuraba en torno a $2 \%$ de Discapacidad intelectual Severa, $43 \%$ de Discapacidad intelectual Moderada, 46\% de Discapacidad intelectual Leve y, 9\% bajo un C. I. de 75 puntos (limítrofes). Un $90 \%$ era de extrema pobreza. El nivel de analfabetismo de los padres correspondía a un 17\% y existía un nivel de cesantía del $24 \%$ entre los padres y apoderados.

A partir de los resultados obtenidos, y tomando en cuenta el bajo nivel socioeconómico de los alumnos, fue posible categorizar a la población estudiantil como una de alto riesgo social y con importante índice de vulnerabilidad.

Para satisfacer uno de los objetivos de la Escuela -"favorecer la integración de los alumnos a la comunidad"- se provee de atención pedagógica individualizada y grupal a los cursos básicos integrados cuyos estudiantes podrán optar a educación básica común después de dos a tres años de preparación. Para los cursos básicos especiales, que atienden a niños con mayor discapacidad intelectual, la propuesta de integración se elabora en torno al Programa de Capacitación Laboral, cuyo objetivo principal es "desarrollar habilidades sociolaborales y, en forma secundaria, técnicas específicas que permitan al alumno insertarse en el mundo del trabajo".

La Escuela había explorado en diversas áreas del Desempeño Ocupacional sin resultados satisfactorios hasta el año 2003 cuando, al sumarse al equipo de trabajo una Terapeuta Ocupacional, se tomó conciencia de la necesidad de realizar cambios profundos en la estructura de los Talleres. Se planteó la necesidad de conformar un equipo interdisciplinario, establecer redes de apoyo comunitario, confeccionar instrumentos de evaluación específicos y colaborar con el hallazgo de puestos de trabajo acordes con las necesidades de cada joven. Además, se proyectó el Programa de Capacitación Laboral como una instancia que promoviera la adquisición de competencias sociales y laborales como claves en el éxito de la inserción sociolaboral. Por último, se proponía la realización de reuniones mensuales de apoderados para abordar temas de su interés que favorecieran la comprensión y ayuda efectiva hacia los escolares.

Para llevar a cabo la reestructuración del Programa Laboral se conformó un equipo interdisciplinario compuesto por Terapeuta Ocupacional, Asistente Social, Jefe Unidad Técnico Pedagógica, Directora del establecimiento y Profesoras de Educación Diferencial- que, a través de un proceso de reflexión, replanteó los Talleres Laborales como un proceso educativo que entrega herramientas para la vida, potenciando habilidades y destrezas para desempeñarse en el ámbito laboral. Al equipo antes descrito se sumó una Psicóloga a partir de marzo de 2005.

Una vez diseñado, el Programa de Capacitación Laboral comienza a funcionar, en forma paulatina, a partir del segundo semestre de 2003. En estos momentos contempla dos Pretallares: uno en el que los alumnos son dependientes para la realización de sus AVD (Actividades de la Vida Diaria) y otro, en el que son independientes; y tres Talleres Laborales. En los Pretalleres los contenidos curriculares se orientan al aprendizaje de lenguaje y matemáticas, y al logro de la máxima autonomía en la ejecución de las AVD. Además, realizan "pasantías" una o dos veces por semana por los diferentes Talleres de la Escuela para promover la familiarización de los alumnos de Pretaller con las actividades propias de cada 
Taller Laboral.

Al cumplir con determinados criterios de egreso los educandos pasan a la etapa de Talleres Laborales, estructurados en torno a un plan común de habilidades socio laborales y a un programa específico de aprendizaje técnico. En esta fase, las visitas a empresas se vinculan con el área temática del Taller y tienen por objeto la observación en terreno de puestos de trabajo y de conductas laborales. Actualmente se cuenta con tres Talleres: Alimentación, Lavandería y Jardinería. Los dos primeros funcionan en dependencias de la Escuela y el otro, en Mundo Granja, programa de la Facultad de Ciencias Veterinarias y Pecuarias de la Universidad de Chile.

Los alumnos que cumplen con los criterios de egreso de los Talleres Laborales son evaluados por el equipo interdisciplinario y por el Profesor a cargo de su Taller para comenzar una Práctica Laboral Comunitaria, en la cual cada uno deberá trabajar en un puesto laboral contactado por la Escuela en condiciones similares a cualquier trabajador. Si la experiencia resulta exitosa se le ayuda a conseguir trabajo en ése u otro lugar; si no lo es, el muchacho reingresa a su Taller. Los estudiantes que, después de ocho años como máximo, no logren estar capacitados para ser incluidos en el campo laboral, serán derivados a Talleres Laborales Protegidos de la comuna o se les asesorará en la formación de famiempresas.

El presente artículo tiene por objeto dar a conocer el Programa de Capacitación Laboral de la Escuela Gabriela Mistral como una alternativa concreta para la integración sociolaboral de personas con discapacidad intelectual. Este proyecto pretende demostrar que -aun cuando los recursos económicos sean escasos y la población beneficiaria provenga de un medio socioeconómico marginal- al conformar un buen equipo interdisciplinario, establecer redes comunitarias adecuadas y elaborar instrumentos de evaluación y seguimiento específicos, es factible reducir las barreras sociales y laborales para las personas con discapacidad intelectual ayudándoles a acceder al mundo laboral bajo mejores condiciones.

\section{- Metodología "}

Para lograr el propósito de capacitación e inserción laboral efectivo, se han tomado en cuenta las necesidades de los alumnos, las sugerencias de los padres y del equipo interdisciplinario con el propósito de desarrollar planes y programas con las estrategias pedagógicas y el sistema de evaluación pertinentes.

Esta metodología, basada fundamentalmente en desarrollar habilidades sociolaborales comunes a cualquier trabajo, pretende, en forma primordial, el crecimiento personal a través del oficio que caracteriza a cada Taller y la inserción en una oferta laboral, según el perfil del puesto de trabajo y de las competencias de cada egresado. Además, la permanencia en el Taller permite el aprendizaje de técnicas específicas y el despliegue de habilidades psicomotoras.

La estructura del Programa considera un plan común y uno complementario para los Pretalleres y un mismo marco curricular para todos los Talleres. El tiempo de permanencia dependerá de los objetivos logrados y de la edad cronológica del alumno. En los Talleres, la permanencia máxima será de 8 años. Cada Taller contempla tres niveles de acuerdo con la adquisición de competencias sociolaborales y al grado de supervisión que se necesite: Formación, Capacitación y Producción. Cada uno sugiere el nivel de integración laboral al que el joven podrá optar: en una empresa, taller protegido, o en un proyecto familiar (famiempresa)

En forma paralela al trabajo cotidiano se realizarán diferentes interacciones con la comunidad: visitas de observación a empresas relacionadas con la temática del Taller, aprendizaje dualy práctica de finalización. Luego del egreso e inserción laboral comienza el período de seguimiento, con un año de 
duración; esta etapa comprende el apoyo del equipo interdisciplinario hacia el alumno y la empresa.

El equipo se ha preocupado también de incluir a la familia en forma permanente, a través de todo el proceso; así, se realizan reuniones mensuales que abordan temáticas de su interés, además de atenciones individuales.

\section{- Pretalleres}

En la actualidad, existe un Pretaller conformado por 15 alumnos, nueve hombres y seis mujeres, que trabajan toda la carga horaria en la Escuela, tienen un sistema de pasantías por los Talleres de oficio una vez a la semana, donde realizan actividades sencillas con el propósito de acercarlos hacia la dinámica de Taller y, de acuerdo con las metas de la capacitación, desarrollar, esencialmente, conductas sociolaborales.

Características de la Puesta en Marcha:

- El equipo interdisciplinario participa desde la disciplina que cada uno representa en la sistematización del programa: se apoya al docente, padres y alumnos y se coordinan las redes de apoyo. Se realizan entrevistas a los jóvenes para determinar intereses, potencial vocacional, y enfrentar las dificultades socioafectivas de quienes lo requieran.

- Se establece un plan educativo individual de acuerdo con necesidades y competencias del alumno.

- La malla curricular tiene un énfasis vocacional y cognitivo para enfrentar en el futuro a los Talleres Laborales, así, el plan de trabajo se aboca a afianzar habilidades sociales basadas en los requerimientos de las conductas sociolaborales. Se promueve también la formación afectiva, moral y sexual.

- Se aplica un plan de aprendizaje de lenguaje y matemáticas como herramientas efectivas en el campo laboral, insistiendo en las habilidades comunicativas, en la escritura funcional, comprensión de información cuantitativa y aplicación de estrategias para resolución de problemas, especialmente aquellos relativos al dinero.

- Se destinan horas para exploración y desarrollo de habilidades manuales, y pasantías en los Talleres de oficio en los cuales los estudiantes se inician en las actividades más elementales.

- Se evalúan conductas observables y posibles de medir en todas las áreas de aprendizaje para calcular el logro de metas concretas y definir la promoción a Talleres Laborales. Las pautas de evaluación aplicadas son:

1. Pauta de evaluación estandarizada: cuadro para la evaluación en el desarrollo social y personal (P.A.C).

2. Pauta de evaluación no estandarizada de las habilidades sociales.

3. Pauta de evaluación de destrezas en el oficio.

4. Pauta de evaluación de plan común.

\section{" Talleres laborales}

Características de la Puesta en Marcha:

- El marco curricular general se basa en el desarrollo de conductas sociolaborales (genéricas para cualquier trabajo, por sobre el oficio en sí mismo) que diferencian los niveles de competencia 
logrados: formación, capacitación y producción. Se establecenobjetivos educacionales de acuerdo con las conductas esperadas en el cumplimiento de funciones y tareas: autonomía en el puesto de trabajo, respeto por los horarios, permanencia en la actividad, rutina de trabajo, secuencia en la realización de las actividades; así como actitud en su relación con el supervisor y sus pares, habilidades de comunicación, disciplina, presentación y autocuidado, cumplimiento de normas de seguridad y prevención de riesgos.

- Se elabora un plan de desarrollo del lenguaje oral y escrito y de matemáticas en torno a tareas laborales y comunicacionales con el propósito de lograr el máximo aprendizaje en el manejo de lectura, escritura y destrezas matemáticas que permitan la menor segregación posible en la inserción laboral. Se utilizan estrategias basadas en portadores de textos y utilización de los elementos lingüísticos y matemáticos relacionados con el campo laboral.

- Se aplican estrategias progresivas de capacitación: los alumnos tienen 15 horas de trabajo insertas en los módulos de trabajo correspondientes a cada Taller, en ellos, son responsables del producto o servicio que se elabora. El profesor entrega las instrucciones y exige las conductas sociolaborales que se requieren de acuerdo con los potenciales, capacidades y ritmo propios de cada discípulo. La rotación de un módulo a otro está determinada por los logros y dificultades que se van produciendo en el proceso.

- Los alumnos no participan de las actividades cotidianas de la escuela con el propósito de desescolarizarlos.

- Cuando el alumno se encuentra en condiciones básicas de interactuar en el campo laboral, se planifica e implementa un sistema de aprendizaje dual en empresas del sector. En esta etapa el joven queda bajo la supervisión diaria del encargado del puesto de trabajo, debiendo cumplir con todas las exigencias y tareas encomendadas. Además, se establece un sistema de supervisión por parte de la Escuela, a cargo de la Terapeuta Ocupacional quien, a su vez, determina un sistema de coordinación durante el proceso y en el momento de evaluar. En algunos casos los alumnos pueden recibir algo de dinero para pagar su movilización y colación. Cuando ya se han cumplido los objetivos específicos de aprendizaje puede regresar al Taller, dejando el puesto para la práctica de otro alumno.

- Se crean instrumentos de evaluación para determinar permanencia en la etapa, paso a la siguiente o egreso. Los instrumentos utilizados comprenden diferentes etapas del proceso y son aplicados por los diferentes profesionales del equipo interdisciplinario.

\section{- Instrumentos de Evaluación .}

1. Pauta de evaluación estandarizada: cuadro para la evaluación en el desarrollo social y personal $\left(\right.$ P.A.C) ${ }^{(*)}$ : Se aplica al inicio del año para obtener información sobre fortalezas y debilidades en las áreas de independencia personal, comunicación, socialización y ocupación que permitan elaborar un plan educativo individual y, al finalizar el año, corroborar los logros obtenidos

2. Pauta de evaluación no estandarizada de las habilidades sociolaborales ${ }^{(*)}$ : Evalúa conductas requeridas en los Talleres Laborales. Se aplica periódicamente obteniendo un resultado mensual, semestral y anual.

3. Pauta de evaluación de destrezas en el oficio ${ }^{(*)}$ : Relacionada con las destrezas y tareas del Taller al cual asisten y categorizadas de acuerdo con los puestos de trabajo, subdivididas en tareas y actividades.

4. Pauta de evaluación de plan común ${ }^{(*)}$ : Según objetivos planteados por alumno, corresponden a los objetivos de lenguaje, matemáticas, orientación, educación física y tecnología.

5. Análisis del puesto de trabajo: Pauta que determina tareas y actividades en cada módulo. Este análisis permite establecer si es pertinente o no para un alumno en particular.

6. Entrevistas individuales: de acuerdo con los requerimientos, y a la etapa que esté cursando, cada estudiante es citado por el equipo interdisciplinario para ser evaluado.

7. Pauta de evaluación multidisciplinaria: Define incorporación a prácticas, determina egreso y/o 
inserción laboral.

( $\left.{ }^{*}\right)$ También se aplica para pretalleres

\section{" Breve descripción de cada Taller Laboral "}

Taller de Jardinería.

El Taller de Jardinería está compuesto por 15 jóvenes, entre 16 y 26 años -11 hombres y 4 mujeres- a cargo de una Educadora Diferencial. Realizan actividades dos días a la semana dentro de la Escuela desarrollando los objetivos del plan común, y durante los tres días restantes trabajan en las parcelas de Mundo Granja , iniciativa pedagógica que se lleva a cabo desde el año 2000 a la fecha, donde cultivan las habilidades sociolaborales y el aprendizaje de la técnica del oficio.

En la actualidad, se realizan agro cultivos en cinco parcelas con cuadrillas de dos a cuatro alumnos. En ellas se han distribuido huertas, invernaderos y cajones con hierbas aromáticas. Al cabo de su cosecha, los productos serán adquiridos por académicos de la Facultad quienes forman parte de un sistema de apadrinamiento de los estudiantes.

Los puestos de trabajo son:

1. Utilización de herramientas y maquinarias: reconocer, trasladar, aprender el uso.

2. Módulo jardines: sembrar, regar, plantar, desmalezar, cortar césped.

3. Módulo huerta: sembrar, plantar, limpiar, regar, cosechar.

4. Módulo invernadero: sembrar, plantar, limpiar, transplantar.

Taller de Alimentación

Se inició en el año 2003 con una cocina y muy pocos interesados. Hoy en día, la Educadora Diferencial tiene a su cargo 15 alumnos, 5 hombres y 10 mujeres entre 16 y 26 años; el $80 \%$ presenta discapacidad intelectual leve a moderada. El Taller funciona en dependencias del colegio y cuenta con un módulo habilitado con artefactos, utensilios y accesorios requeridos para el oficio. Las actividades se organizan destinando tres días de la semana para la preparación de alimentos, y dos, al plan común.

Los puestos de trabajo son:

1. Encargado de aseo y ornato: lavar, secar, guardar y ordenar.

2. Preparador de alimentos: seleccionar, lavar, limpiar, picar, rallar y otros.

3. Asistente de cocina: asistir al preparador de alimentos.

4. Encargado de despensa: clasificar, guardar provisiones, revisar fechas.

5. Organización, presentación ornamental de productos y atención al público.

Taller de Lavandería

Está compuesto por 15 alumnos, de 16 a 26 años -11 hombres y cuatro mujeres- a cargo de una Educadora Diferencial. El 90 \% de los alumnos presenta discapacidad intelectual moderada a severa. Se perfila como un futuro Taller Protegido debido a las características de sus participantes y por las ganancias que genera. Funciona en dependencias del colegio con una sala habilitada con maquinaria y artefactos indispensables para el oficio. Tres días a la semana las actividades están destinadas a 
lavandería, secado y planchado de plumones y ropa en general, y dos, al plan común.

Los puestos de trabajo son:

1. Recepción y despacho: atender público al recibir y entregar las prendas.

2. Pre lavado a máquina: recibir las prendas, cargar la lavadora y programarla.

3. Pre lavado a mano y lavado a máquina: clasificar, remojar y escobillar.

4. Centrifugado y secado: clasificar, programar la secadora y colgar las prendas.

5. Planchado: clasificar, planchar, doblar, empaquetar y etiquetar.

6. Insumos y almacenaje: identificar las necesidades, almacenar la mercadería.

\section{Cuadro resumen del Proceso Educativo de la Escuela Gabriela Mistral:}

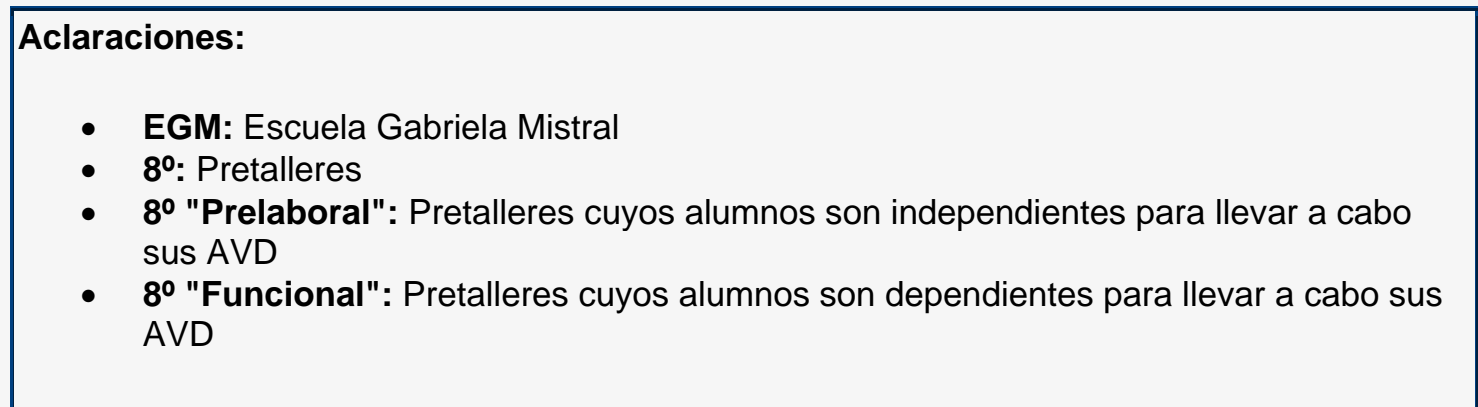

\section{- Resultados "}

El Programa de Capacitación Laboral ha obtenido resultados exitosos en diferentes áreas:

\section{Funcionamiento de la Escuela Gabriela Mistral:}

- Creación de un equipo interdisciplinario:

Permite tener una visión integral de la capacitación laboral a través de un proceso de reflexión continua y establecimiento de roles complementarios.

La incorporación de la Terapeuta Ocupacional al equipo permitió tener una visión más profunda de la capacitación sociolaboral.

- Trabajo técnico pedagógico:

La reestructuración del Programa de Capacitación e Inserción Laboral requirió la creación de planes y programas acordes con los nuevos planteamientos, y el desarrollo de instrumentos evaluativos que ofrezcan una imagen real de los aprendizajes de los alumnos.

- Apoyo a los Profesores:

El trabajo conjunto con el equipo de gestión, en reuniones individuales y grupales de análisis del funcionamiento de los Talleres y de apoyo en la aplicación de material evaluativo, otorga valor a la tarea 
desempeñada y una sensación de amparo ante la nueva propuesta educativa.

- Desarrollo de los alumnos:

Adquisición de nuevas habilidades psicomotoras y asentamiento de conocimientos anteriores, junto con aprendizaje en terreno de habilidades sociolaborales y técnicas de un oficio.

Incremento de la autonomía personal: al constatar logros individuales en el quehacer diario y, al percibir un refuerzo positivo y un apoyo adecuado por parte de sus padres.

El análisis de la jornada de trabajo desarrollado el día anterior permite tomar conciencia de las dificultades, así como de la forma adecuada para enfrentar los problemas diarios que se les puedan presentar.

Conocimiento de la realidad laboral a través de las visitas de observación permitiéndoles asomarse a un mundo hasta ahora desconocido.

Tomar conciencia de su capacidad de obtener una remuneración por el trabajo desarrollado, lo que refuerza lo que la Escuela les proporciona en el sentido de adquirir seguridad en sí mismos y autovalorarse.

- Incorporación activa de padres y apoderados:

La creación de la Escuela para Padres les ha hecho sentir apoyo por parte de la Escuela para la educación de sus hijos y les ha proporcionado herramientas para enfrentar mejor las situaciones conflictivas. En forma secundaria, les ha provisto de conocimientos para mejorar su propia situación y la de su familia.

Se les incorpora a la rutina diaria de los Talleres a través de visitas programadas para conocer y apoyar lo que hacen sus hijos.

\section{Apertura hacia la Comunidad:}

- Taller de Jardinería (Trabajo en Mundo Granja):

Mundo Granja ha adquirido un compromiso con la discapacidad al permitir a la Escuela trabajar en sus dependencias en forma indefinida y al abrirse a nuevas experiencias con personas discapacitadas sensorialmente.

Trabajo interinstitucional fluido entre dos instancias educativas.

Inserción de dos alumnos en práctica remunerada en Mundo Granja y de un tercer alumno incorporado en forma indefinida.

Venta de los productos del Taller de jardinería en Expo- Rural Internacional en stand ofrecido por Mundo Granja y en la comunidad educativa.

- Taller de Lavandería:

A través del lavado de ropa de la comunidad aledaña, principalmente del comercio del sector (peluquerías, restaurantes, empresas, Club de Leones, etc.) ha logrado dar a conocer y enseñar a valorar 
a las personas con discapacidad, conformando una red de apoyo para la escuela.

- Taller de Alimentación :

Venta de productos a la población educativa, vecinos, empresas de la comuna, Club de Leones de La Cisterna y Mundo Granja.

Actualmente cuatro alumnos se encuentran haciendo aprendizaje dual en el Supermercado Lider S.A. del sector, donde asisten por media jornada, dos veces a la semana, a trabajar guiados por un padrino en los sectores de Pastelería y Panadería.

\section{- Discusión "}

El programa de Talleres Prelaborales y Laborales de la Escuela Gabriela Mistral es una propuesta de trabajo, factible de ser replicada, que pretende lograr la integración socio laboral a través de la capacitación a los alumnos en técnicas determinadas en cada uno de los Talleres, y proveer un espacio para la adquisición de habilidades sociolaborales que son, en definitiva, las que permitirán una inserción laboral exitosa. Por eso, durante la puesta en marcha se ha dado especial énfasis a esta última área, confeccionando evaluaciones específicas que han ayudado a una valoración objetiva con posibilidades de visualizar el desempeño individual y grupal experimentado en términos mensuales, semestrales y anuales. Así, la técnica en sí pasa a segundo plano en términos de evaluación, pero continúa teniendo importancia cotidiana si se toma en cuenta que los alumnos deben adquirir habilidades y destrezas que les permitan una mayor y mejor productividad.

Lo anterior se sustenta en que si los jóvenes consiguen desarrollar condiciones que permitan un buen desempeño en relaciones interpersonales -con sus pares y jefes-, manejo adecuado de la frustración, aceptación de críticas, seguimiento de instrucciones, etc., serán capaces de conseguir y mantener un puesto de trabajo, aun cuando deban realizar actividades diferentes de las que aprendieron en los Talleres Laborales, pues sería más rápido desarrollar tareas específicas.

La experiencia ganada hasta ahora con la planificación y puesta en marcha del Programa de Pretalleres y Talleres Laborales en la Escuela Gabriela Mistral permite asignar al trabajo de un equipo interdisciplinario un rol fundamental en el éxito de la empresa. La multivisión de las problemáticas que van surgiendo permite un análisis exhaustivo en búsqueda de las soluciones más adecuadas; la división de tareas, por su parte, ayuda a un trabajo ágil y eficiente y, por último, permite a cada uno de los involucrados una participación activa y directa en la toma de decisiones, así como colaborar en la adquisición de un compromiso real con el proyecto institucional.

En este contexto, el rol del Terapeuta Ocupacional como parte del equipo interdisciplinario de educación es valorado en la medida en que su aporte teórico se traduce en contribuciones innovadoras para el quehacer diario de los escolares. Las tareas fundamentales han sido: participación en reuniones periódicas de planificación y evaluación; elaboración de documentos en conjunto con la Unidad Técnico Pedagógica de acuerdo con la realidad local y las necesidades que han ido surgiendo en el desarrollo de la experiencia, por ejemplo, pautas de evaluación de cada alumno para determinar ingreso al Taller Laboral que consideran intereses, habilidades y percepción del Profesor a cargo; estudios de cada puesto de trabajo, realizados en conjunto con los Profesores del establecimiento de cada uno de los Talleres, lo que permite orientar a cada joven en los pasos por seguir en cada actividad; análisis abreviado de cada puesto de trabajo para evaluar aquellos que en la comunidad sean acordes con las necesidades particulares del estudiante y de la Escuela. Esto, en conjunto con el desarrollo de pautas de evaluación mensuales y semestrales diseñadas para cada Taller Prelaboral y Laboral, ha resultado clave 
en el buen funcionamiento del programa.

En la etapa de prácticas laborales el papel del Terapeuta Ocupacional se ha planteado como un mediador entre las partes involucradas, empresa - escuela - alumno - familia, para facilitar los procesos de cada una.. Este rol se postula como interdependiente del resto del equipo con el fin de dividir tareas y optimizar los resultados.

De acuerdo con la experiencia obtenida hasta ahora, surge la necesidad de incorporar la disciplina de Terapia Ocupacional desde los inicios de la formación en la Escuela especialmente para intervenir en la malla curricular planificando objetivos tendientes a afianzar habilidades sociales precursoras de conductas sociolaborales.

La Asistente Social interviene en las familias cuando no hay compromiso o, por el contrario, existe resistencia a la autonomía del educando; además, realiza atenciones individuales y visitas domiciliarias.

La tarea del Educador Diferencial en los Talleres Laborales constituye el cimiento sobre el cual se sustenta el éxito en la preparación laboral. Debe sistematizar el funcionamiento de los Talleres, desescolarizando la dinámica de trabajo y optimizando la ejecución de actividades: utilizar el tiempo en forma eficiente, organizar rutinas laborales, establecer y mantener normas consistentes en la convivencia y en la prevención de riesgos, dominar el oficio o buscar y actualizar conocimientos, tener claro cuáles son las tareas y secuencias de los módulos, disponer de un ambiente de trabajo organizado, así como de espacios y recursos, entregar instrucciones claras y precisas, tomar en cuenta la diferencia en las capacidades de los educandos, transmitirles expectativas sobre sus posibilidades de aprendizaje (utilizar estrategias de retroalimentación), actuar con asertividad y empatía en todos los momentos del aprendizaje, abordar la evaluación de acuerdo con las pautas e instrumentos elaborados para el Taller, recoger evidencias operacionales de logros en los objetivos, manejar el nivel de competencia de cada escolar, ser capaz de proponer discípulos para las prácticas en el campo laboral, y para su egreso, y, por último, participar en la etapa de seguimiento una vez que el alumno se ha insertado laboralmente, manejando información sobre fallas y éxitos del egresado.

El equipo, en conjunto, organiza e interviene en los Talleres para padres, que han tenido muy buena acogida, tanto por ellos como por toda la Escuela, lo que se ve ratificado por el alto porcentaje de asistencia y participación durante la sesión.

En forma paralela se ha llevado a cabo el trabajo interdisciplinario al interior de la Escuela y con Mundo Granja, que pretende, por un lado, ofrecer a los jóvenes del Taller de Jardinería la posibilidad de aprender en terreno habilidades sociolaborales y aspectos técnicos del trabajo agrícola, junto con permitir, a través de prácticas laborales, la puesta en escena de los conocimientos adquiridos. Por otro lado, este trabajo conjunto ha logrado ampliar los horizontes educacionales de la institución universitaria hacia jóvenes con discapacidad intelectual, dándoles la oportunidad de interactuar con personal técnico y administrativo en un entorno diferente al acostumbrado, y permitiendo a Mundo Granja conocer la realidad de personas con esta discapacidad, ampliando su público objetivo. De este modo, han incluido en su agenda de actividades el trabajo con niños con discapacidades sensoriales. En este sentido el programa ha establecido un puente entre una institución tradicional de educación superior como es la Universidad de Chile, a través de uno de los programas de la Facultad de Ciencias Veterinarias y Pecuarias - Mundo Granja -, y el mundo de la discapacidad intelectual, representado por 15 jóvenes de la Escuela Gabriela Mistral.

Cabe destacar la evolución que ha tenido el programa en cuanto a personas involucradas de Mundo Granja: en una primera etapa el contacto era con personal técnico y administrativo, hoy, en cambio, se suma la participación de docentes de la Facultad por medio del apadrinamiento de educandos y de su producción, lo que induce a un compromiso personalizado de los profesores hacia "su ahijado" y, por otro lado, promueve el sentido de responsabilidad y compromiso junto con la estimulación del sentido de pertenencia, de autoestima y el trabajo en equipo en pro de un objetivo común, por parte de cada 
alumno.

Otra forma en que los Talleres Laborales han ayudado a una apertura de la Escuela hacia la Comunidad local es a través de la comercialización de la producción: El Taller de Jardinería participó con un stand en la Feria Internacional Expo- Rural 2003 y vende su producción por medio de Mundo Granja y de la Escuela. Los Talleres de Lavandería y Alimentación benefician con sus servicios a los vecinos y a la comunidad escolar. Con las ganancias los Talleres colaboran con su propia puesta en marcha por medio de la adquisición de nuevos insumos y, cuando hay excedentes, alcanza para entregar un incentivo monetario a sus participantes.

Las reuniones mensuales de apoderados, en las que el equipo aborda diferentes temas propuestos por ellos mismos, han tenido muy buena acogida ya que, por primera vez, se les da la opción de elegir temas por analizar respecto a la condición y educación de sus hijos. Así, se han abordado temas sobre autonomía personal, sexualidad, drogadicción, violencia intrafamiliar, alternativas de inclusión al mundo laboral, entre otros.

El desarrollo de habilidades sociolaborales concluye en una práctica final, donde el alumno se proyecta en el campo laboral. En la mayoría de los casos, la práctica se continúa con la inserción definitiva en el mismo puesto de trabajo, ante lo cual, el empleador considera al "alumno" como un trabajador en condiciones de ser contratado.

En este momento surge como obstáculo legal la pensión que reciben los alumnos como beneficio estatal: pensión asistencial de invalidez que consiste en la entrega mensual vitalicia de una cantidad de dinero que, en la actualidad, corresponde a \$37.849 por tratarse de personas en situación de extrema pobreza y que presentan discapacidad intelectual. Así, la realidad laboral del sistema económico de libre mercado que rige para todos los chilenos pone en peligro el Programa de Capacitación e Inserción Sociolaboral de la Escuela en su última etapa: los alumnos deben optar: o renuncian a su pensión en favor de un contrato, lo que implica perder la seguridad económica que otorga la pensión, o establecen uno formal que, por un lado, provee un mayor ingreso, pero por otro, puede terminar por cualquier motivo y en cualquier momento. De esta manera, la Escuela cumple con el proceso completo, pero ve peligrar la factibilidad de la inserción laboral definitiva ya que debe ser el joven egresado y su familia los que tomen la decisión final.

Otro de los obstáculos que deben enfrentar las personas con discapacidad intelectual corresponde a las obligaciones legales y tributarias que deben ejercer, especialmente las relacionadas con contratos, trámites provisionales y tributarios. Las falencias cognitivas limitan el desarrollo de habilidades lingüísticas y matemáticas, y reducen su capacidad de tomar decisiones y de reconocer situaciones que vayan en desmedro de su integridad. De ahí que sean individuos socialmente vulnerables.

Asimismo, la experiencia ha demostrado que la persona con discapacidad intelectual sufre la estigmatización de la sociedad chilena; por consiguiente la población que aborda la Escuela es doblemente segregada: no solo se le discrimina por sufrir una discapacidad, sino que, también, por provenir de un nivel socioeconómico de extrema pobreza, lo que disminuye las posibilidades efectivas de inserción sociolaboral.

Otro factor que se debe tener en cuenta para la participación activa de las personas con discapacidad en la sociedad, es que una modalidad importante de inserción laboral lo constituyen los Talleres Protegidos: instancias queacogen a jóvenes y adultos con déficit intelectual que requieren apoyo continuo de un instructor del oficio; en su mayoría, realizan tareas simples y específicas de una cadena de producción o servicio. Estos Talleres no reciben subvención estatal alguna, sino que se mantienen por autofinanciamiento, compitiendo con la oferta y demanda del mercado según la calidad y valor del producto. Esto implica dimisiones en quienes han tomado estas iniciativas, dejando a sus integrantes con la única opción de permanecer en el hogar. 
A partir de lo anterior es posible concluir que en Chile la integración laboral de personas con discapacidad intelectual y de muy escasos recursos enfrenta falencias importantes en el sistema que dificultan la puesta en marcha y el éxito de los esfuerzos realizados localmente. Así, el marco legal, el financiamiento de proyectos que no solucionan la continuidad laboral de quienes participan, y la falta de apoyo e inversión económica para abordar de manera óptima este sector de la sociedad, son factores que deben abordarse desde un punto de vista comunitario por tratarse de una responsabilidad compartida entre todos los habitantes del país: la inclusión efectiva de personas con alguna discapacidad.

\section{- Agradecimientos "}

El buen funcionamiento del Programa de Capacitación Laboral no podría haberse concretado si no se hubiese contado con el apoyo permanente de la Administración de la Escuela Gabriela Mistral, con el trabajo consistente del Equipo Interdisciplinario y con la labor abnegada de los profesores de cada Pretaller y Taller. Sin duda, el aporte de Don Nelson Matamala al transportar a los jóvenes, y el de Don Carlos Ortiz ayudando en la infraestructura y puesta en marcha de los Talleres, han sido de vital importancia.

El Taller de Jardinería no podría funcionar en óptimas condiciones, si no fuera por la fundamental ayuda de Mundo Granja -Programa de la Facultad de Ciencias Veterinarias y Pecuarias de la Universidad de Chile, de sus coordinadores: Don José Antonio Villarroel y la Srta. Macarena Ocariz. El refuerzo cotidiano de su personal técnico ha contribuido especialmente en la adquisición de técnicas específicas para cada puesto de trabajo. Para el desarrollo del programa de apadrinamiento resulta clave el patrocinio y compromiso de los docentes de la Facultad.

Por último, se desea agradecer a los vecinos de las comunas El Bosque y La Cisterna por su interés en ayudar a los Talleres adquiriendo sus productos.

\section{- Referencias "}

Dado que el presente artículo tiene por objetivo dar a conocer una experiencia práctica, se estimó que no era imprescindible recurrir a una revisión bibliográfica exhaustiva. Los dos apoyos teóricos a los que se recurrió fueron:

- Los trabajos publicados por el Prof. Miguel Ángel Verdugo A., M. A. quien ha desarrollado importantes aportes en varias líneas de investigación: Retraso Mental. Calidad de Vida, Habilidades Sociales, Empleo, Evaluación de Programas. Discapacidad, Maltrato y Actitudes.

- Otro precedente importante fue el Estudio de Investigación "Situación de la Formación Laboral de la Educación Especial" realizado por el Ministerio de Educación de chile (MINEDUC) durante el año 2002. 